\title{
On the Explosion Geometry of Red Supergiant Stars
}

\author{
Douglas C. Leonard ${ }^{1}$, Luc Dessart ${ }^{2}$,Giuliano Pignata ${ }^{3}$, D. John \\ Hillier $^{4}$, George G. Williams ${ }^{5}$, Paul S. Smith ${ }^{6}$, Harish Khandrika ${ }^{1,7}$, \\ Christopher Bilinski $^{6}$, Nhieu Duong ${ }^{1}$, Kelsi Flatland ${ }^{1}$, Luis Gonzalez ${ }^{1}$, \\ Jennifer L. Hoffman ${ }^{8}$, Chuck Horst ${ }^{1}$, Leah Huk ${ }^{8}$, Peter Milne ${ }^{6}$, \\ Alisa A. Rachubo ${ }^{1}$ and Nathan Smith ${ }^{6}$ \\ ${ }^{1}$ San Diego State University, ${ }^{2}$ U. Nice Sophia-Antipolis, ${ }^{3}$ U. Andres Bello, ${ }^{4}$ U. Pittsburgh, \\ ${ }^{5}$ MMT Observatory, ${ }^{6} \mathrm{U}$. Arizona, ${ }^{7}$ Space Telescope Science Institute, ${ }^{8}$ U. Denver
}

\begin{abstract}
We present the observed "continuum" levels of polarization as a function of time for four well-observed Type II-Plateau supernovae (SNe II-P; Fig. 1), the class of SNe decisively determined to arise from red supergiant stars (Smartt 2009). All four objects show temporally increasing degrees of polarization through the end of the photospheric phase, with some exhibiting early-time polarization that challenge existing models (e.g., Dessart and Hillier 2011) to reproduce. A fundamental ejecta asymmetry is present in this photometrically diverse sample of type II SNe, and it probably takes different forms (e.g., ${ }^{56} \mathrm{Ni}$ blobs/fingers, large scale deformation). We acknowledge support from NSF grants AST-1009571 and AST-1210311.
\end{abstract}

Keywords. Supernovae: individual (SN 2004dj, SN 2008bk, SN 2012aw, SN 2013ej)

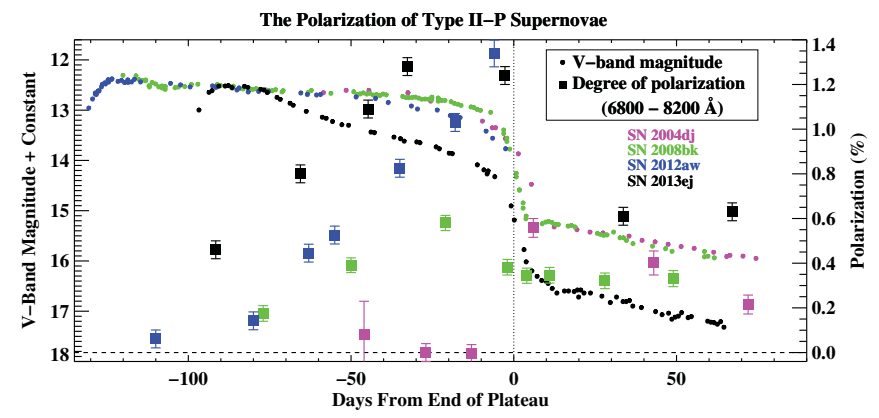

Figure 1. Photometry data have been shifted vertically for clarity, and polarization data have been corrected for interstellar polarization. Data sources: SN 2004dj (Leonard et al. 2006); SN 2008bk (Leonard et al. 2012); SN 2012aw (photometry: Dall'Ora et al. 2014; polarimetry: This work); SN 2013ej (photometry: Richmond 2014 - note the relatively steep plateau decline compared to the other objects in this sample; polarimetry: This work).

\section{References}

Dall'Ora, M., et al. 2014, ApJ, 787, 139

Dessart, L. \& Hillier, D. J. 2011, MNRAS, 415, 3497

Leonard, D. C., et al. 2006, Nature, 440, 505

Leonard, D. C., Dessart, L., Hillier, D. J., \& Pignata, G. 2012, in: J. Hoffman, B. Whitney, \& J. Bjorkman (eds.), Stellar Polarimetry (New York, AIP), 1429, 204

Richmond, M. W. 2014, JAAVSO, 42, 333

Smartt, S. J. 2009, ARAA, 47, 63 